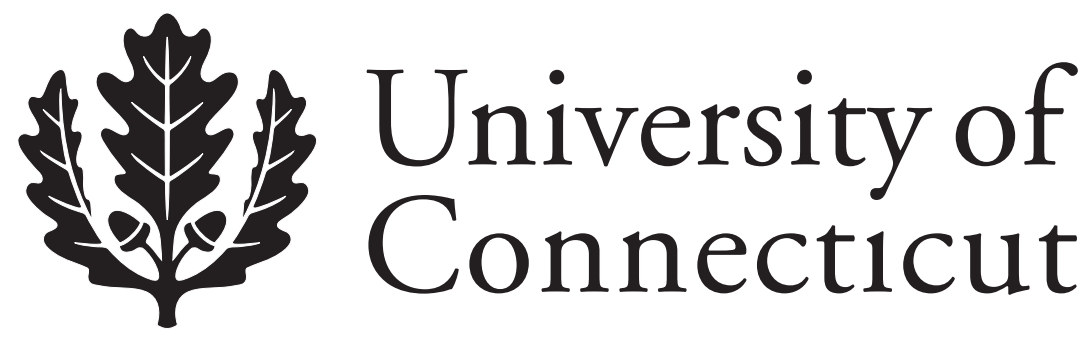

Department of Economics Working Paper Series

\title{
Three-agent Peer Evaluation
}

Vicki Knoblauch

University of Connecticut

Working Paper 2008-28

August 2008

341 Mansfield Road, Unit 1063

Storrs, CT 06269-1063

Phone: (860) 486-3022

Fax: (860) 486-4463

http://www.econ.uconn.edu/

This working paper is indexed on RePEc, http://repec.org/ 


\begin{abstract}
I show that every rule for dividing a dollar among three agents impartially (so that each agent's share depends only on her evaluation by her associates) underpays some agent by at least one-third of a dollar for some consistent profile of evaluations. I then produce an impartial division rule that never underpays or overpays any agent by more than one-third of a dollar, and for most consistent evaluation profiles does much better.
\end{abstract}

Journal of Economic Literature Classification: D70, D63

Keywords: division function, impartial, consensual 


\title{
Three-agent Peer Evaluation
}

\author{
Vicki Knoblauch \\ Department of Economics \\ University of Connecticut
}

\begin{abstract}
I show that every rule for dividing a dollar among three agents impartially (so that each agent's share depends only on her evaluation by her associates) underpays some agent by at least one-third of a dollar for some consistent profile of evaluations. I then produce an impartial division rule that never underpays or overpays any agent by more than one-third of a dollar, and for most consistent evaluation profiles does much better.
\end{abstract}

Journal of Economic Literature Classification Codes: D70, D63

Keywords: division function, impartial, consensual

Correspondence:

Vicki Knoblauch

Department of Economics

University of Connecticut

Storrs, CT

06269-1063

USA

phone: 8604869076

fax: 8604864463

e-mail: vicki.knoblauch@uconn.edu 


\section{Introduction.}

A principal hires three agents to work together on a project. The principal agrees to pay the team a fixed sum when the project is completed. It must be decided how to allocate the fixed sum among the three agents. The only information available consists of agents' evaluations of the relative contributions of their partners. For example, agent 1 might report that agent 3's contribution was five times as great as agent 2's; agent 2 might report that agent 1's contribution was seven times as great as agent 3's; and agent 3 might report that agent 1's contribution was 35 times as great agent 2's. A rule is needed in the form of a division function that will input the peer evaluations and output the amounts the agents will receive.

Three desirable properties of a division function are exactness, impartiality and consensuality. A division function is exact if it always outputs amounts that add up to the fixed sum; impartial if the amount paid to an agent is independent of that agent's report; consensual if the amounts paid to the agents respect the peer evaluations whenever those evaluations are consistent. (In the example above the evaluations are consistent since $5 \times 7=35$. A consensual division rule would assign agents 1,2 and 3 the fractions $35 / 41$, $1 / 41$ and 5/41 of the fixed sum, respectively.)

Unfortunately, de Clippel et al. (2008) showed that in the three-agent case a division function cannot be exact, impartial and consensual. There remains the problem of choosing a division function that satisfies at most two of the three desirable properties. We require exactness since in our version of the problem the principal is committed to paying out a fixed sum. We will investigate exact, impartial division functions, which eliminates strategic considerations. In what follows we will determine the extent to which an exact, impartial division function must deviate from consensuality. We will adopt a worst case measure of deviation from consensuality. More exactly, we define the deviation from consensuality of a division function to be the supremum of the absolute value of the difference between the amount assigned to an agent by the given division function and the amount assigned by a consensual division function. Here the supremum is taken over all three agents and over all consistent peer evaluations. We find that the minimum deviation 
from consensuality for an exact, impartial division function is $1 / 3$. We also produce an exact, impartial division function with that minimal deviation. This division function is also symmetric with respect to agents, and each agent's payoff is increasing in each of her evaluations by her partners.

Dividing a resource of a fixed size among a fixed number of agents is a problem that can take on a number of forms. Thomson (2003) surveys the literature pertaining to the resolution of conflicting claims over a resource. There is also a literature on cake cutting and pie cutting that focuses on fair division of a divisible good, parts of which are valued differently by different parties. Brams et al. (2006) provide a recent contribution to this literature. de Clippel et al. (2008) raised the question at hand, that of dividing a dollar using a rule that depends only on agents' evaluations of their associates. They showed that in the three-agent case there can be no exact, impartial, consensual rule. They also explored exact, impartial, consensual rules for more than three agents. Tideman and Plassman (2008) studied the extent to which the unique impartial, consensual division rule fails to be exact in the three-agent case. Our study can be thought of as complementary to the three-agent section of their paper, since we study the extent to which all impartial, exact division rules fail to be consensual in the three-agent case.

The result establishing $1 / 3$ as the minimal deviation from consensuality of a threeagent exact, impartial division function, and discussion of an example are contained in Section 3, which is preceeded by a preliminary section and followed by some concluding remarks.

\section{Preliminaries.}

Three agents complete a project and submit peer evaluations $r=\left(r^{1}, r^{2}, r^{3}\right)$ where agent $i$ 's evaluation is $r^{i}=\left(r_{j, k}^{i}, r_{k, j}^{i}\right) \in[0,+\infty] \times[0,+\infty]$ with $\{i, j, k\}=\{1,2,3\}, j<k$ and $r_{k, j}^{i}=\frac{1}{r_{j, k}^{i}}$, including the conventions $0=\frac{1}{+\infty}$ and $+\infty=\frac{1}{0}$. The evaluation $r^{i}=$ $\left(r_{j, k}^{i}, r_{k, j}^{i}\right)$ indicates that agent $i$ asserts that agent $j$ 's contribution to the project was $r_{j, k}^{i}$ times as great as agent $k$ 's contribution.

Payments are assigned to the agents according to the division function $f(r)=\left(f_{1}(r), f_{2}(r), f_{3}(r)\right)$ where $0 \leq f_{i}(r)<+\infty$ for $i \in\{1,2,3\}$. Now we set the fixed 
payment sum at 1 for the sake of simplicity. The division function is exact if $f_{1}(r)+$ $f_{2}(r)+f_{3}(r)=1$ for every peer evaluation profile $r$. The division function $f$ is impartial if for every $i \in\{1,2,3\}$ agent $i$ 's payment $f_{i}$ does not depend on $r^{i}$. Then we can write $f_{i}(r)=f_{i}\left(r_{k, i}^{j}, r_{j, i}^{k}\right)$ for $(i, j, k) \in T=\{(1,2,3),(2,3,1),(3,1,2)\}$.

Peer evaluations are consistent if $r_{23}^{1} r_{31}^{2}=r_{21}^{3}$ or if one of $r_{23}^{1}$ and $r_{31}^{2}$ is 0 and the other is $+\infty$. The division function is consensual if it respects consistency, that is, if $f_{1}(r)+f_{2}(r)+f_{3}(r)=1$ and $f_{i}(r)=r_{i, j}^{k} f_{j}(r)$ for $\{i, j, k\}=\{1,2,3\}$ both hold when evaluations are consistent, with the convention $a=+\infty \times 0$ for all $a \in[0,1]$.

\section{Exact and Impartial Division Functions.}

As mentioned above, de Clippel et al. (2008) showed that for three agents a division function cannot be exact, impartial and consensual. We will now investigate exact, impartial division functions to determine the extent to which they must deviate from consensuality.

Suppose $f$ is an exact, impartial division function. Then for $(i, j, k) \in T=\{(1,2,3)$, $(2,3,1),(3,1,2)\}$ and any peer evaluation profile $r$,

$$
f_{i}\left(r_{k, i}^{j}, r_{j, i}^{k}\right)+f_{j}\left(r_{i, j}^{k}, r_{k, j}^{i}\right)+f_{k}\left(r_{j, k}^{i}, r_{i, k}^{j}\right)=1
$$

Setting $r_{j, k}^{i}=1$, for $(i, j, k) \in T$ and $\left(r_{k, i}^{j}, r_{j, i}^{k}\right) \in[0,+\infty] \times[0,+\infty]$

$$
f_{i}\left(r_{k, i}^{j}, r_{j, i}^{k}\right)=1-f_{j}\left(r_{i, j}^{k}, 1\right)-f_{k}\left(1, r_{i, k}^{j}\right)
$$

From (2) we see that if $f$ is an exact, impartial division function, then each $f_{i}$ is additively separable. We also have the following.

Proposition 1. If $f$ is an exact, impartial division function, then $\sum_{i=1}^{3} f_{i}(1,1)=1$.

Proof: Let $r_{j, k}^{i}=r_{k, i}^{j}=r_{i, j}^{k}=1$ for $(i, j, k)=(1,2,3)$. The conclusion follows from (1). 
Proposition 2. If $f$ is an exact, impartial division function and $a_{i}, b_{i} \in[0,+\infty]$ for $i \in\{1,2,3\}$, then $\sum_{i=1}^{3} f_{i}\left(a_{i}, 1\right)+\sum_{i=1}^{3} f_{i}\left(1, b_{i}\right) \leq 3$.

Proof: For $(i, j, k) \in T$, from $(2) f_{i}\left(\frac{1}{b_{k}}, \frac{1}{a_{j}}\right)=1-f_{j}\left(a_{j}, 1\right)-f_{k}\left(1, b_{k}\right)$. Then summing over $T$

$$
\sum_{i=1}^{3} f_{i}\left(\frac{1}{b_{k}}, \frac{1}{a_{j}}\right)=3-\sum_{j=1}^{3} f_{j}\left(a_{j}, 1\right)-\sum_{k=1}^{3} f_{k}\left(1, b_{k}\right)=3-\sum_{i=1}^{3} f_{i}\left(a_{i}, 1\right)-\sum_{i=1}^{3} f_{i}\left(1, b_{i}\right)
$$

By the definition of a division function, the left side of the first equation is at least zero.

Proposition 3. If $f$ is an exact, impartial division function and $a_{i}, b_{i} \in[0,+\infty]$ for $i \in\{1,2,3\}$, then $\sum_{i=1}^{3} f_{i}\left(a_{i}, b_{i}\right) \leq 2$.

Proof: By (2), for $(i, j, k) \in T$,

$$
\begin{aligned}
f_{i}\left(a_{i}, b_{i}\right) & =1-f_{j}\left(\frac{1}{b_{i}}, 1\right)-f_{k}\left(1, \frac{1}{a_{i}}\right) \\
& =1-\left(1-f_{k}(1,1)-f_{i}\left(1, b_{i}\right)\right)-\left(1-f_{i}\left(a_{i}, 1\right)-f_{j}(1,1)\right)
\end{aligned}
$$

Then summing over $T$,

$$
\sum_{i=1}^{3} f_{i}\left(a_{i}, b_{i}\right)=3-6+2 \sum_{i=1}^{3} f_{i}(1,1)+\sum_{i=1}^{3} f_{i}\left(a_{i}, 1\right)+\sum_{i=1}^{3} f_{i}\left(1, b_{i}\right)
$$

By Propositions 1 and 2 $\sum_{i=1}^{3} f_{i}\left(a_{i}, b_{i}\right) \leq 3-6+2+3=2$.

Corollary 1. If $f$ is an exact, impartial division function, then for some $i \in\{1,2,3\}$, $f_{i}(a, b) \leq 2 / 3$ for all $a, b \in[0,+\infty]$.

Proof: Fix $\epsilon>0$ and for $i \in\{1,2,3\}$ choose $a_{i}, b_{i} \in[0,+\infty]$ such that $f_{i}\left(a_{i}, b_{i}\right) \geq$ $\sup f_{i}-\epsilon=\sup \left\{f_{i}(x, y): x, y \in[0, \infty]\right\}-\epsilon$. By Proposition $3, \sum_{i=1}^{3} f_{i}\left(a_{i}, b_{i}\right) \leq 2$. Therefore, $f_{i}\left(a_{i}, b_{i}\right) \leq 2 / 3$ for some $i$. For this $i$, sup $f_{i} \leq \frac{2}{3}+\epsilon$. Therefore, for some $i$, $\sup f_{i} \leq 2 / 3$.

Definition. The deviation from consensuality of a division function $f$ is $D(f)=$ $\sup \left\{\left|f_{i}(r)-\frac{1}{1+r_{k, i}^{j}+r_{j, i}^{k}}\right|: r\right.$ is a peer evaluation profile and $\left.(i, j, k) \in T\right\}$.

We so define deviation from consensuality because de Clippel et al. (2008) showed that the unique impartial, consensual division function is $f_{i}(r)=\frac{1}{1+r_{k, i}^{j}+r_{j, i}^{k}}$ for $(i, j, k) \in T$. 
Corollary 2. The deviation from consensuality of an exact, impartial division function is at least $1 / 3$.

Proof. Suppose $f$ is an exact, impartial division function. Then $D(f) \geq\left|f_{i}(0,0)-\frac{1}{1+0+0}\right|$ for $i=1,2,3$. By Corollary 1 , there is an $i$ such that $f_{i}(0,0) \leq \frac{2}{3}$. Then $D(f) \geq\left|\frac{2}{3}-1\right|=$ $\frac{1}{3}$.

To see that the bounds in Proposition 2 and 3 and Corollary 1 and especially Corollary 2 are tight, consider the following example.

Example 1. For $(i, j, k) \in T$, let

$$
F_{i}\left(r_{k, i}^{j}, r_{j, i}^{k}\right)=\frac{1}{6}\left(2+\frac{1-r_{k, i}^{j}}{1+r_{k, i}^{j}}+\frac{1-r_{j, i}^{k}}{1+r_{j, i}^{k}}\right)
$$

We have chosen this function because it is the simplest division function satisfying the properties we are about to establish for it. Clearly, $F$ is impartial. That $F$ is exact follows from the fact that for $(i, j, k) \in T, \frac{1-r_{j, k}^{i}}{1+r_{j, k}^{2}}+\frac{1-r_{k, j}^{i}}{1+r_{j, k}^{i}}=0$ (recall that $r_{j, k}^{i}=\frac{1}{r_{k, j}^{i}}$ ). For this example, $F_{i}(0,1)=F_{i}(1,0)=\frac{1}{2}$ and $F_{i}(0,0)=\frac{2}{3}$ for $i \in\{1,2,3\}$. In addition, $F$ is symmetric with respect to agents (by which is meant $F_{i}(a, b)=F_{j}(a, b)=F_{j}(b, a)$ for $i, j \in\{1,2,3\}$ and $a, b \in[0,+\infty])$; and $F_{i}$ is decreasing in $r_{k, i}^{j}$ and $r_{j, i}^{k}$ for $(i, j, k) \in T$.

Finally, we will show that $D(F)=\frac{1}{3}$. Let $E(x, y)=\frac{1}{6}\left(2+\frac{1-x}{1+x}+\frac{1-y}{1+y}\right)-\frac{1}{1+x+y}$ so that $D(F)=\sup |E|$ on $[0,+\infty] \times[0,+\infty]$. In fact $\sup |E|=\max |E|$ since $E$ is continuous on $[0,+\infty] \times[0,+\infty]$. We will first work on the boundary of $[0,+\infty] \times[0,+\infty]$. There, $E(x, 0)=\frac{1}{6}\left(3+\frac{1-x}{1+x}\right)-\frac{1}{1+x}=\frac{x-1}{3(1+x)}$ so that $E(x, 0)$ is increasing from $E(0,0)=-\frac{1}{3}$ to $E(+\infty, 0)=\frac{1}{3}$. Next $E(x,+\infty)=\frac{1}{6}\left(2+\frac{1-x}{1+x}-1\right)=\frac{1}{3(1+x)}$ so that $E(x,+\infty)$ is decreasing from $E(0,+\infty)=\frac{1}{3}$ to $E(+\infty,+\infty)=0$. On the boundary of $[0,+\infty] \times[0,+\infty]$, $\max (|E|)=\frac{1}{3}$ and the maximum occurs at $(0,0),(+\infty, 0)$ and $(0,+\infty)$.

In the interior of $[0,+\infty] \times[0,+\infty]$, setting $\frac{\partial E}{\partial x}=\frac{\partial E}{\partial y}=0$ and solving, $x_{0}=y_{0}=$ $1+\sqrt{3}$. For the only candidate for a maximum of $|E|$ in the interior, $E\left(x_{0}, y_{0}\right)=.024$.

In summary, the deviation from consensuality for $F$ is $\frac{1}{3}$ and occurs when agent $i$ is rated $r_{k, i}^{j}=r_{j, i}^{k}=0$ in which case agent $i$ is underpaid compared to a consensual division, or when agent $i$ is rated $r_{k, i}^{j}=0$ and $r_{j, i}^{k}=+\infty$, or $r_{k, i}^{j}=+\infty$ and $r_{j, i}^{k}=0$ in which case agent $i$ is overpaid compared to a consensual division. 


\section{Concluding Remarks.}

Notice that for our division function $F$, extreme overpayment or underpayment compared to consensual payment occurs only for extreme peer evaluations. For example, the most extreme underpayment of $\frac{1}{3}$ occurs only when an agent is rated $+\infty$ compared to both players, and the most extreme overpayment of $\frac{1}{3}$ occurs only in the even more unusual case when an agent is rated 0 compared to one associate and $+\infty$ compared to another.

On the other hand, for the less extreme consistent evaluation profile $\left(r_{23}^{1}, r_{31}^{2}, r_{12}^{3}\right)=$ $\left(\frac{1}{2}, 4, \frac{1}{2}\right)$ the exact, impartial division function of Example 1 overpays agent 1 by $3 \frac{1}{2}$ cents compared to the impartial, consensual division function, overpays agent 2 by $4 \frac{3}{4}$ cents and underpays agent three by $8 \frac{1}{4}$ cents. 


\section{References}

1. Brams, S. J., Jones, M. A., and Klamler, C. (2006). Better ways to cut a cake: Notices of the American Mathematical Association, 53, 1314-1321.

2. de Clippel, G., Moulin, H., and Tideman, N. (2008). Impartial division of a dollar: Journal of Economic Theory, 139, 176-191.

3. Thomson, W. (2003). Axiomatic and game-theoretic analysis of bankruptcy: Mathematical Social Sciences, 45, 249-297.

4. Tideman, T. N., and Plassmann, F. (2008). Paying the partners: Public Choice, 136, $19-37$. 